\title{
FoxE1 expression is regulated by Sox9 in thyroid follicular cells
}

\section{Arístides López-Márquez, Carlos Carrasco-López and Pilar Santisteban \\ Instituto de Investigaciones Biomédicas "Alberto Sols" CSIC-UAM. Madrid. Spain.}

\section{INTRODUCTION}

Sox9 is a transcription factor of the HMG box DNA binding family involved in the development of endoderm-derivedorgans and the maintenance of the stem/progenitor cells in several tissues. Preliminary results from our laboratory have shown that Sox 9 is expressed in the thyroid gland and its regulation is under the control of TSH and TGF $\beta$. In this work we studied the role of Sox9 in the thyroid cells differentiation and its relationship with the other thyroid transcription factors, mainly with FoxE1.

\section{MATERIALS AND METHODS}

-Cell systems: Rat thyroid follicular cells PCCl3 maintained in Coon's medium $6 \mathrm{H}$ (5\% Donor serum, +TSH, +IGF-1). Hela cells grown in DMEM medium supplemented with Fetal Bovine Serum at $10 \%$.

-DNA binding Activity: Electrophoretic Mobility Shift Assay

-Protein Detection: Western-Blot

-mRNA detection: RT-qPCR

-Transfection assays: Determination of the luciferase activity of the construction pGL3-promRatSox9 and pGI3-promFoxe1 were transfected in PCCl3. Co-

transfections assays were performed with different promoter constructions and expression vectors of Pax8, TTF1 and Sox9.

-Inhibition of Sox9 expression: Speccific Sox9 siRNA was used to downregulate the expression of Sox9 in $\mathrm{PCCl} 3$ cells.

\section{RESULTS}

1) Sox9 binds to its consensus DNA sequence in rat FoxE1 promoter

a)

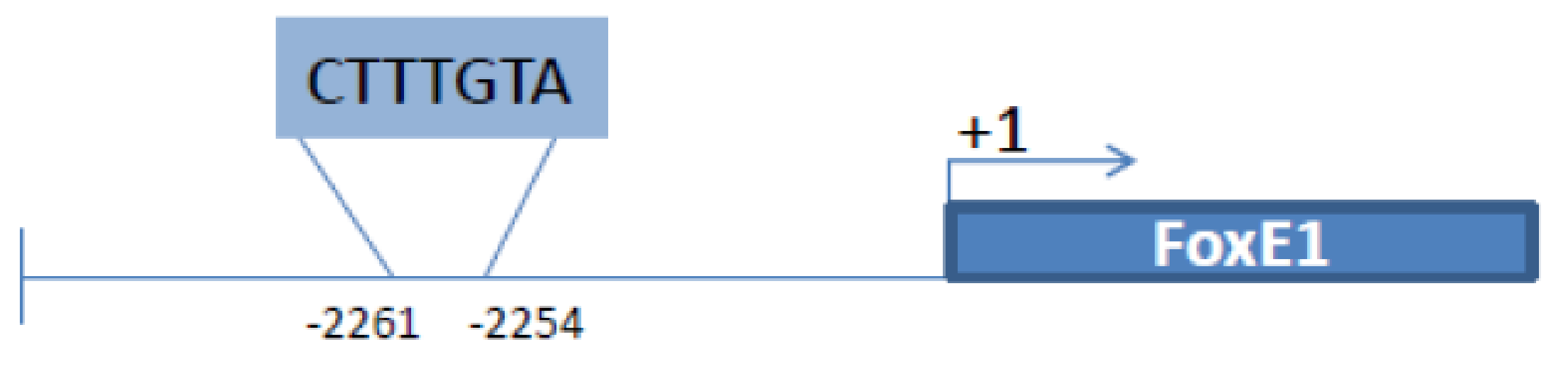

b)

'-TCATGACCTTTGTATTAATC-3 $\begin{array}{lllll}1 & 2 & 3 & 4 & 5\end{array}$

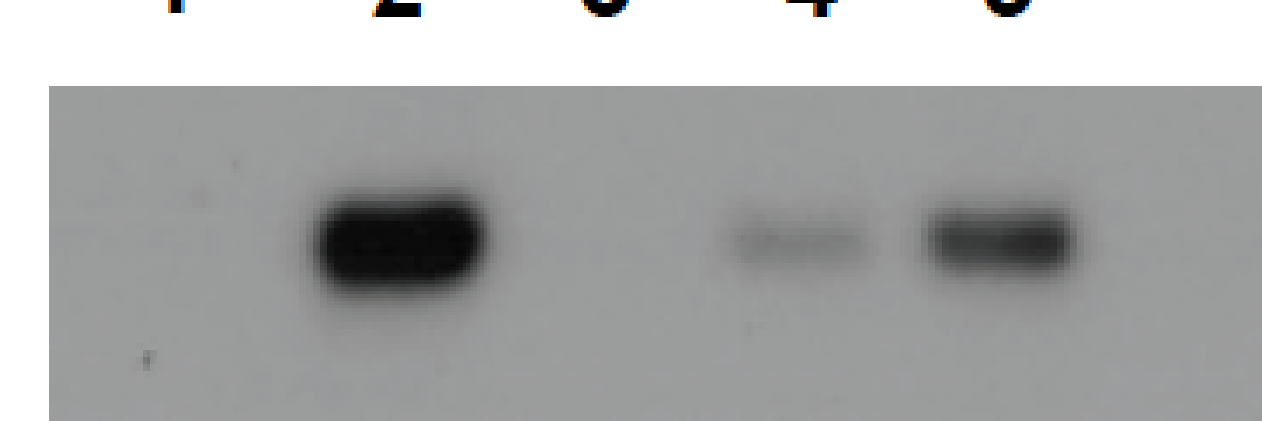

Fig.1: (a) Schematic representation of the Sox9 binding site in the rat FoxE1 promoter (b)Sox9 binding activity to the FoxE1 rat promoter was determined by EMSA. The probe was incubated without (lane 1) or with $5 \mu \mathrm{g}$ of Sox9 purified protein (lanes 2-5) Competition was performed with a 100 -fold molar excess of unlabeled related (NR; lane 4) or unrelated (R;lane 5 ) oligonucleotide.

2) Sox9 induces the transcriptional activity of FoxE1 promoter while FoxE1 inhibits the Sox9 promoter

a)

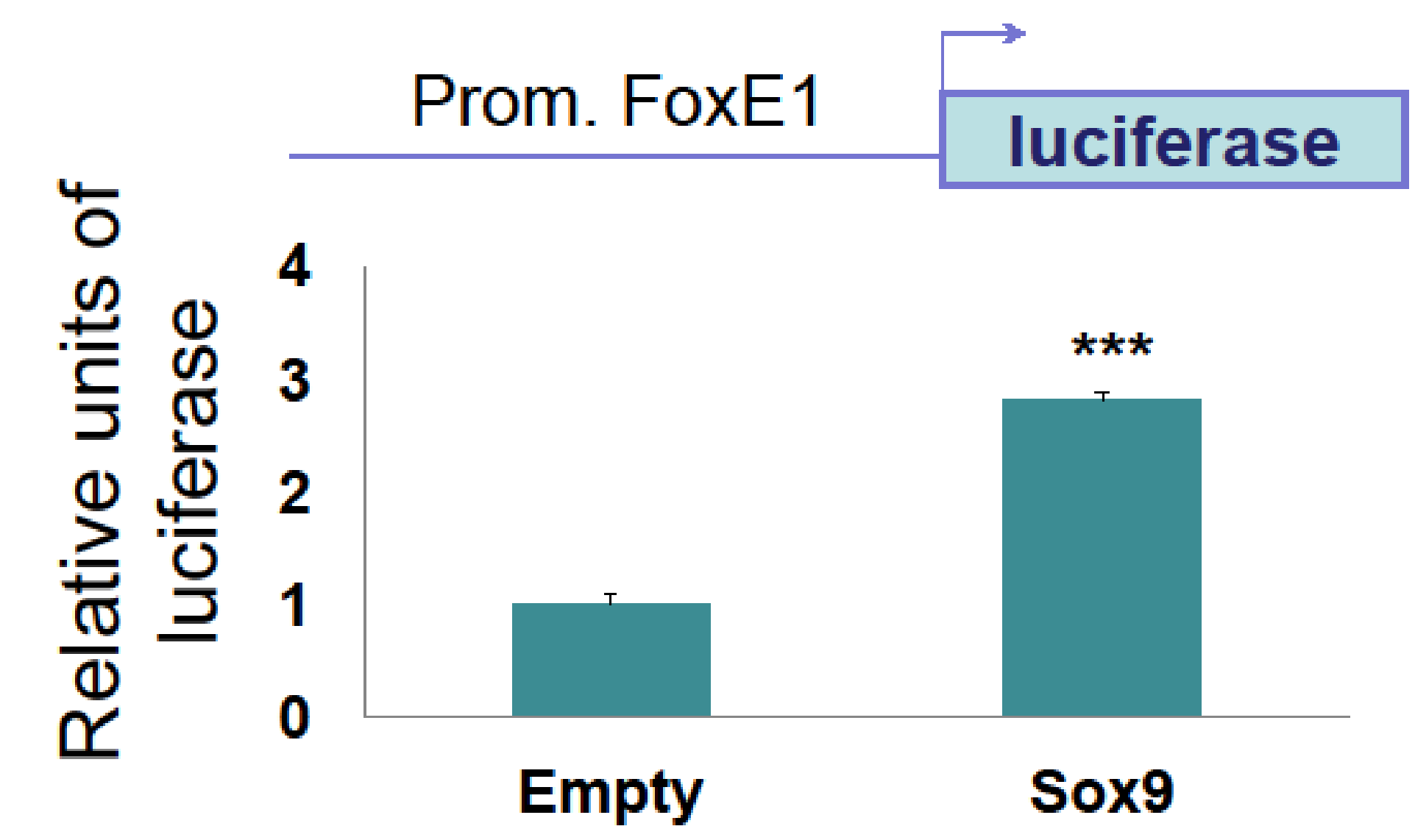

b)

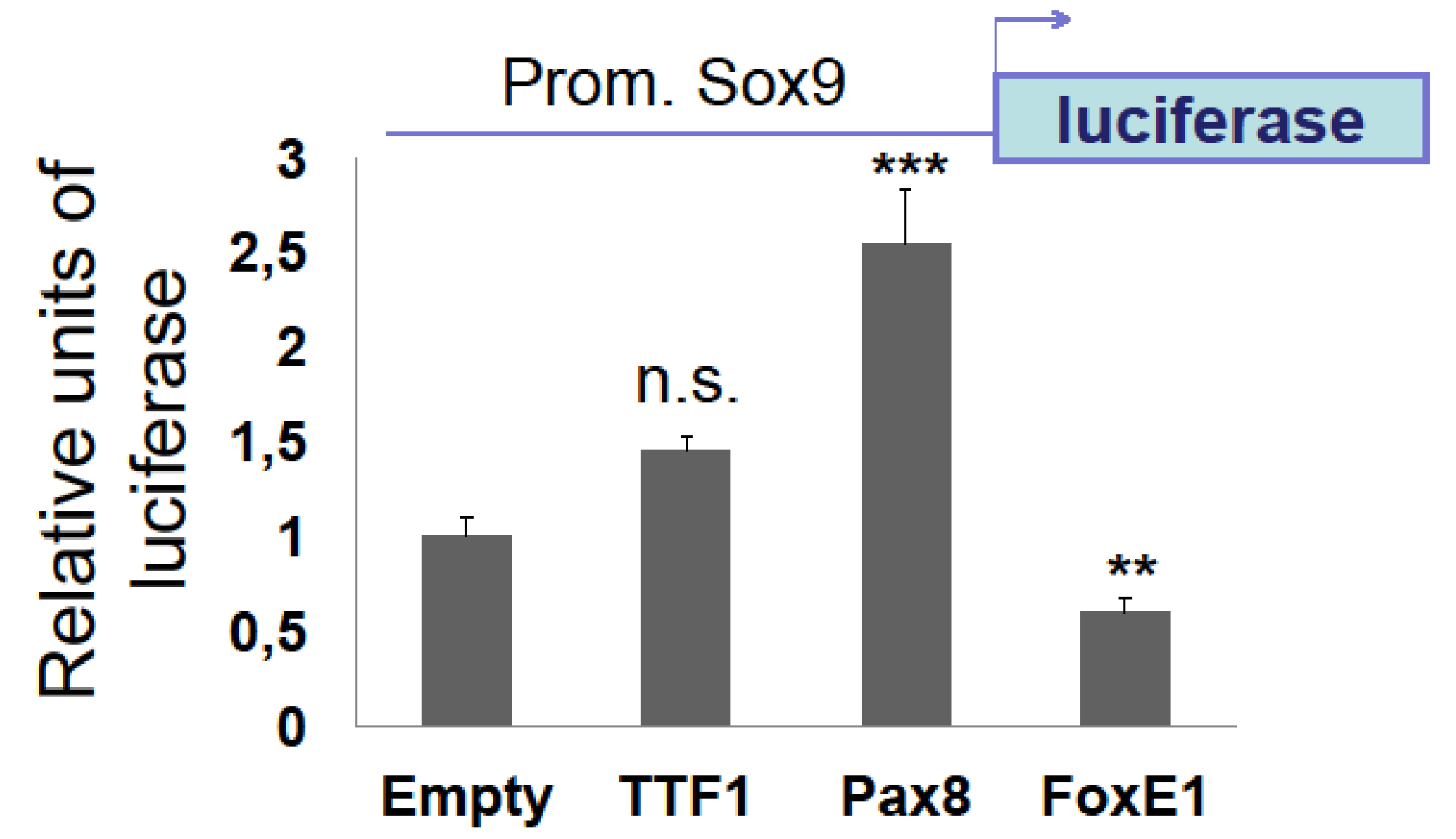

Fig.2: (a) Co-transfecction assays in Hela cells with pGI3-promRatFoxE1 and Sox9 expression vector. (b)Co-transfection assay in Hela cells with pGL3-promRatSox9 and TTF1, Pax8 and FoxE1 expression vectors.

3) Inhibition of Sox9 led to a decrease in the expression levels of FoxE1

a)

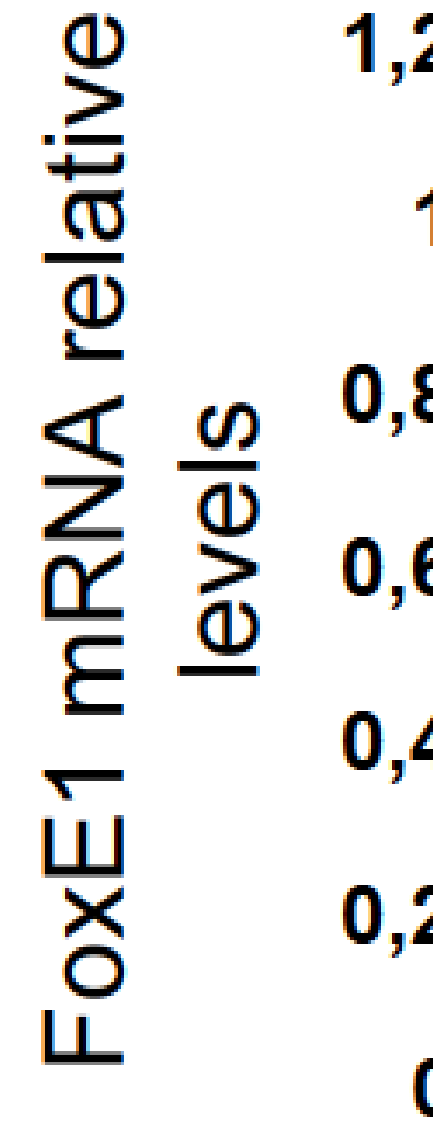

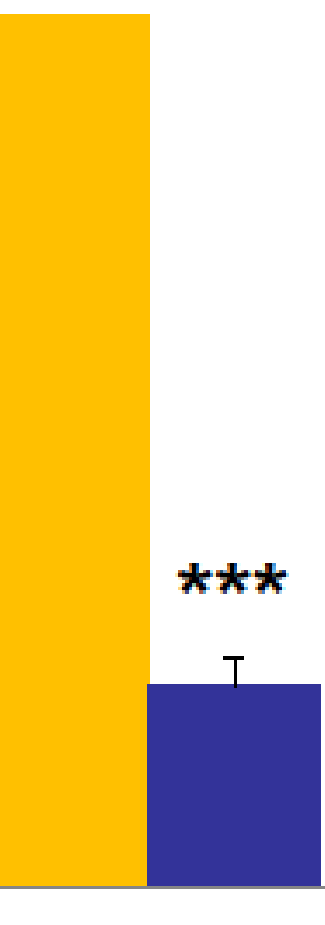

$16 \mathrm{~h}$

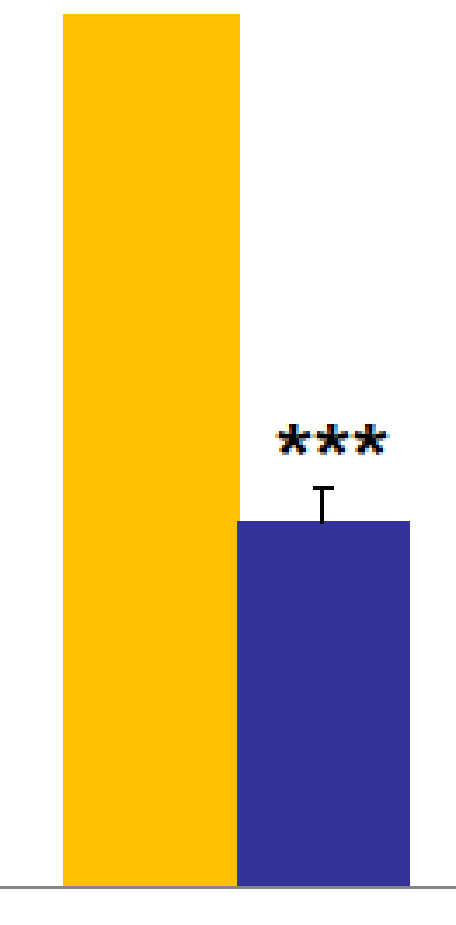

b)

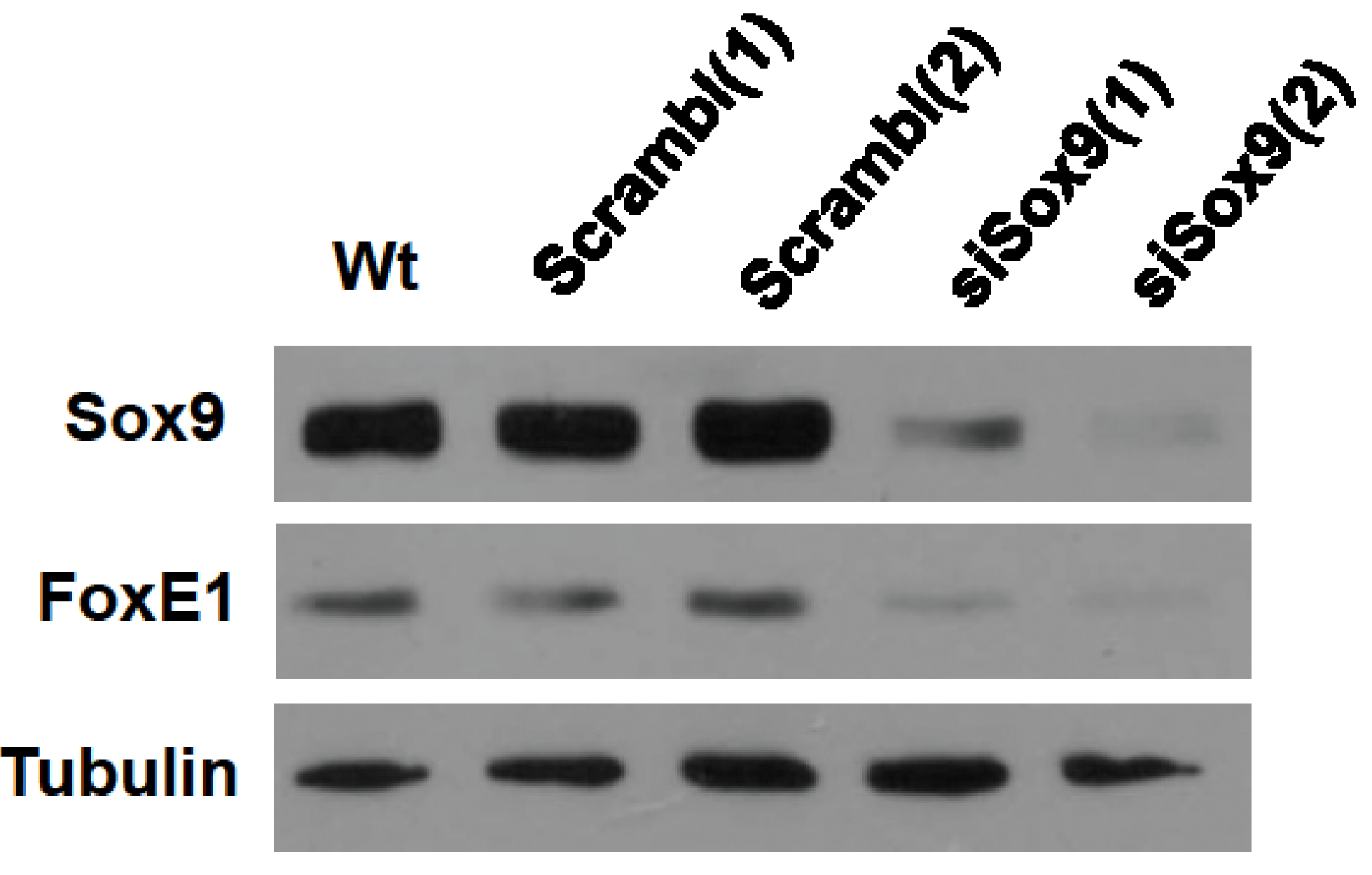

Fig.3: (a) RT-qPCR of FoxE1 mRNA levels of PCCI3 cells transfected at different times with a siRNA specific for Sox9. (b) Western-Blot of Sox9 and FoxE1 protein levels in PCCI3 48 hours after transfection with a siRNA specific for Sox 9 .

\section{CONCLUSIONS}

- Sox9 binds to its consensus sequence in the FoxE1 promoter

- Sox9 induces the expression of FoxE1 thyroid transcription factor, while FoxE1 inhibits Sox9 expression

- Pax8 and TTF1 are involved in the expression control of Sox9

\section{DISCUSSION}

These results demonstrate the involvement of Sox9 in the pathophysiology of the thyroid and its involvement in the regulation of thyroid differentiation program, something hitherto unknown, and open a new field of study in the development and differentiation of the thyroid gland.

\section{e}

European Society of Endocrinology 